\title{
Experimental investigation of the parallel structure of fluctuations in the scrape-off layer of Alcator C-Mod
}

O. Grulke

Max-Planck-Institute for Plasma Physics, Greifswald Branch, EURATOM

Association, Greifswald, Germany

E-mail: grulke@ipp.mpg.de

\section{J.L. Terry, I. Cziegler, and B. LaBombard}

Plasma Science and Fusion Center, Massachusetts Institute of Technology, Cambridge, USA

\section{O.E. Garcia}

Department of Physics and Technology, University of Troms $\varnothing$, Norway

PACS numbers: $47.27,52.30,52.35 . \mathrm{R}$ 


\section{Introduction}

The anomalous transport in magnetically confined plasmas is known for a long time to be driven by turbulent fluctuations $[1,2]$. The paradigm for this fluctuation-induced transport is the advection of density fluctuations within turbulent potential eddies [3]. In the last decade improved spatiotemporal diagnostics revealed a more detailed picture of the transport mechanism in the plasma Scrape-Off-Layer (SOL), i.e. the coherent radial propagation of large-amplitude turbulent plasma pressure structures, commonly called blobs [4-6]. This finding has been shown to be not limited to fusion devices, but is a generic feature of turbulence, occurring also in plasmas with pure toroidal geometry $[7$, $8]$ and even in homogeneously magnetized plasmas [9, 10]. They represent intermittent transport events, which carry particles and heat radially outwards thereby effecting key reactor issues like first wall erosion, helium removal and divertor heat load. Blob transport has been suggested as a process contributing to radial transport of poloidal momentum [11], supporting the formation of stationary shear layers [12].

Although the underlying dynamics for the blob formation and evolution differ with magnetic geometry, the blob propagation perpendicular to the ambient magnetic field is well understood: The self-consistent perpendicular electric field associated with the blob pressure gives rise to a radially outward $E \times B$ motion of the blob [6]. In toroidal geometry measurements have given evidence that the electric field is mainly due to interchange dynamics, which results in a self-consistent potential with a poloidal phase shift of $\pi / 2$ between the blob's plasma pressure and potential $[13,7,8]$. Numerical simulations using this approach give good agreement with the experimental results [14$16,11]$. However, due to the limited diagnostic access, the blob structure parallel to the magnetic field in SOL plasmas remains an open issue.

Blobs at the outboard midplane are known to exist as long filament structures with $k_{\perp} / k_{\|} \gg 1$ [13]. Experimentally it was shown that the topology and propagation properties of fluctuation structures close to (but still outside and above) the X-point resemble the midplane blobs in many aspects [17], although some discrepancies are still outstanding in this regard [18]. Close to the X-point numerical simulations suggest that the strong magnetic shear leads to a de-correlation of fluctuations before they reach the divertor $[19,20]$. Several experimental investigations have focused on the characterization of fluctuations parallel to the magnetic field. In the JET tokamak significant correlation amplitudes of $\approx 40 \%$ in fluctuations of the ion saturations current as measured by Langmuir probes have been observed over parallel connections lengths up to $L=66 \mathrm{~m}$, however these fluctuation structures did not pass through the region of strong magnetic shear at the X-point [21]. Measurements on the ASDEX tokamak revealed qualitatively similar results although a connection along the magnetic field passing close to the X-point was established in this case [22]. However, those studies used only point-to-point connections along the field, which are very sensitive to the actual magnetic field geometry. In recent camera imaging measurements at NSTX, striations at the divertor plate similar to blob filament events have been directly observed 
[23] suggesting that filaments might extend to the divertor plate despite the strong magnetic shear near the X-point. In TFTR the fluctuation filaments were found to extend to the favorable magnetic curvature region [24]. Despite the generally high correlation of fluctuations along the magnetic field, the deduced parallel wavenumber differs significantly between the individual studies ranging from flute-like perturbations in JET and W7-AS $[21,25]$ to wavenumbers much smaller than expected for resistive ballooning modes [24].

The present paper addresses the parallel structure of fluctuations in the SOL of Alcator C-Mod from the outboard midplane to the divertor plate just outside of the lower X-point. The main difference from previous investigations is that a twodimensional imaging diagnostic, resolving in the radial and poloidal dimensions, is used at the outboard midplane to measure fluctuations. This makes the cross-correlation of spatially separated fluctuations much less sensitive to the actual magnetic geometry and allows accurate evaluation of the peak of the correlation function. In Sec. 2 the diagnostic setup, the plasma configurations, and experimental techniques are presented. The results of the correlation analyses are compiled in Sec. 3 and the parallel fluctuation velocity is compared to a basic dispersion relation in Sec. 4. There follows an analysis of the parallel wavelength, Sec. 5 , before the results are summarized and discussed in Sec. 6.

\section{Experimental Setup and Diagnostics}

All experiments described here were done in Ohmic L-mode Deuterium plasmas in lower single null configuration with Greenwald densities $n / n_{\mathrm{GW}}$ in the range from 0.1 to 0.4 . A reciprocating probe, entering the plasma from the outboard side above the midplane, measures time-averaged radial SOL profiles from evaluation of the probe characteristics. The key diagnostic tools for turbulent fluctuation measurements are a combination of

\begin{tabular}{lccc} 
parameter & Configuration 1 & Configuration 2 & Configuration 3 \\
\hline toroidal magnetic field $B_{t}$ & $5.4 \mathrm{~T}$ & $3.60-3.62 \mathrm{~T}$ & $3.58-3.59 \mathrm{~T}(4.63 \mathrm{~T})$ \\
plasma current $I_{P}$ & $600 \mathrm{kA}$ & $674-728 \mathrm{kA}$ & $763-793 \mathrm{kA}(978-1012 \mathrm{kA})$ \\
density $\bar{n}$ & $1 \cdot 10^{20} \mathrm{~m}^{-3}$ & $0.7-0.9 \cdot 10^{20} \mathrm{~m}^{-3}$ & $1.2-1.5 \cdot 10^{20} \mathrm{~m}^{-3}$ \\
Greenwald density $n / n_{G W}$ & $25 \%$ & $8-38 \%$ & $10-19 \%$ \\
outer gap & $1.5 \mathrm{~cm}$ & $0.8 \mathrm{~cm}$ & $1.3 \mathrm{~cm}$ \\
$q_{95}$ & 5.5 & $3.25-3.55$ & $3.0-3.16$
\end{tabular}

electric probes and a spatiotemporal Gas-Puff-Imaging (GPI) diagnostic as depicted in Fig. 1a. The GPI setup consists of a localized Deuterium or Helium gas puff on the outboard midplane $[5,26]$. The $\mathrm{D}_{\alpha}$ or He I $(587.6 \mathrm{~nm})$ emission from the puff is observed by a two-dimensional array of $9 \times 10$ views coupled to fast avalanche photodiodes (APD) [27], which span $3 \mathrm{~cm}$ radially and $3.5 \mathrm{~cm}$ vertically The position of the LCFS is chosen in a way that the total field-of-view (f.o.v.) covers mainly the radial SOL width. In 
comparison to measurements done using fast framing cameras, the photodiodes have a much higher sensitivity and faster frequency response. The sampling frequency of $f_{A P D}=2 \mathrm{MHz}$ allows for real-time observations of the spatiotemporal dynamics of turbulent fluctuations over a wide range of wavenumbers [27], albeit with decreased spatial resolution within the limited f.o.v. The photodiode system views toroidally, i.e. at $\mathrm{a} \approx 8^{\circ}$ angle with respect to the local magnetic field. To get information on the parallel structure of the blob filaments two different sets of electric probes are used, which are toroidally and poloidally separated from the views of the GPI. For these experiments specific magnetic configurations are chosen such that the near-midplane GPI f.o.v. is connected to the probe diagnostics along the magnetic field. This is realized using three configurations with rotational transforms, characterized by $q_{95}$, that are appropriate for the magnetic connection. The extended f.o.v. of the GPI allows for some variation in $q_{95}$ for magnetic mapping between the GPI and any one probe. Table 2 compiles the main discharge parameters. (The "outer gap" is the radial distance between the LCFS and the outboard limiter at the midplane.) The time traces of $B_{t}, I_{p}$, and $n_{e}$ of the respective configurations are compiled in Fig. 2, where the shaded area indicates the time within the shot, during which data are taken.

The poloidal projections of the GPI viewing array and the utilized probes are shown in Fig. 1b. A vertically reciprocating multi-tip probe in the magnetic shear region outside and somewhat above the lower X-point, approximately on the opposite side of the device (toroidally separated from the GPI by $198^{\circ}$ ) is used to measure plasma fluctuations in Configuration 1. The probe tips are constantly biased to measure ion saturation current fluctuations $I_{i, s a t}$ and floating potential fluctuations $\Phi_{\text {float }}$, respectively.

In addition to this vertically reciprocating probe, two other probes embedded in

the tiles of the outer divertor are used in Configurations 2 and 3 to measure $I_{i, s a t}$ and to cross-correlate those signals with the intensity fluctuations in the GPI. These two divertor-probes are also toroidally separated from the GPI by $\approx 198^{\circ}$. One of the divertor-probes ("divertor-probe 9") is at a height a few millimeters above that of the Xpoint and $\approx 10 \mathrm{~cm}$ radially outside it. The other ("divertor-probe 10 ") is approximately $1 \mathrm{~cm}$ above the X-point and further into the common-flux SOL than divertor-probe 9. Any fluctuations propagating between the GPI view and the divertor probes sample more of the high-shear region outboard of the X-point than those propagating between the GPI view and the vertically reciprocating probe.

\subsection{Configuration 1}

This configuration provides a magnetic mapping from the GPI array of views to the vertically reciprocating probe. A toroidal magnetic field of $B_{t}=5.4 \mathrm{~T}$ and a plasma current of $I_{P}=600 \mathrm{kA}$ is chosen, yielding $q_{95}=5.5$. In Fig. $1 \mathrm{~b}$ the magnetic mapping of the probe plunge into the GPI f.o.v., as calculated from the EFIT equilibrium reconstruction, is shown, demonstrating that a large portion of the probe plunge is indeed mapped into the GPI f.o.v.. The average connection length along the magnetic 


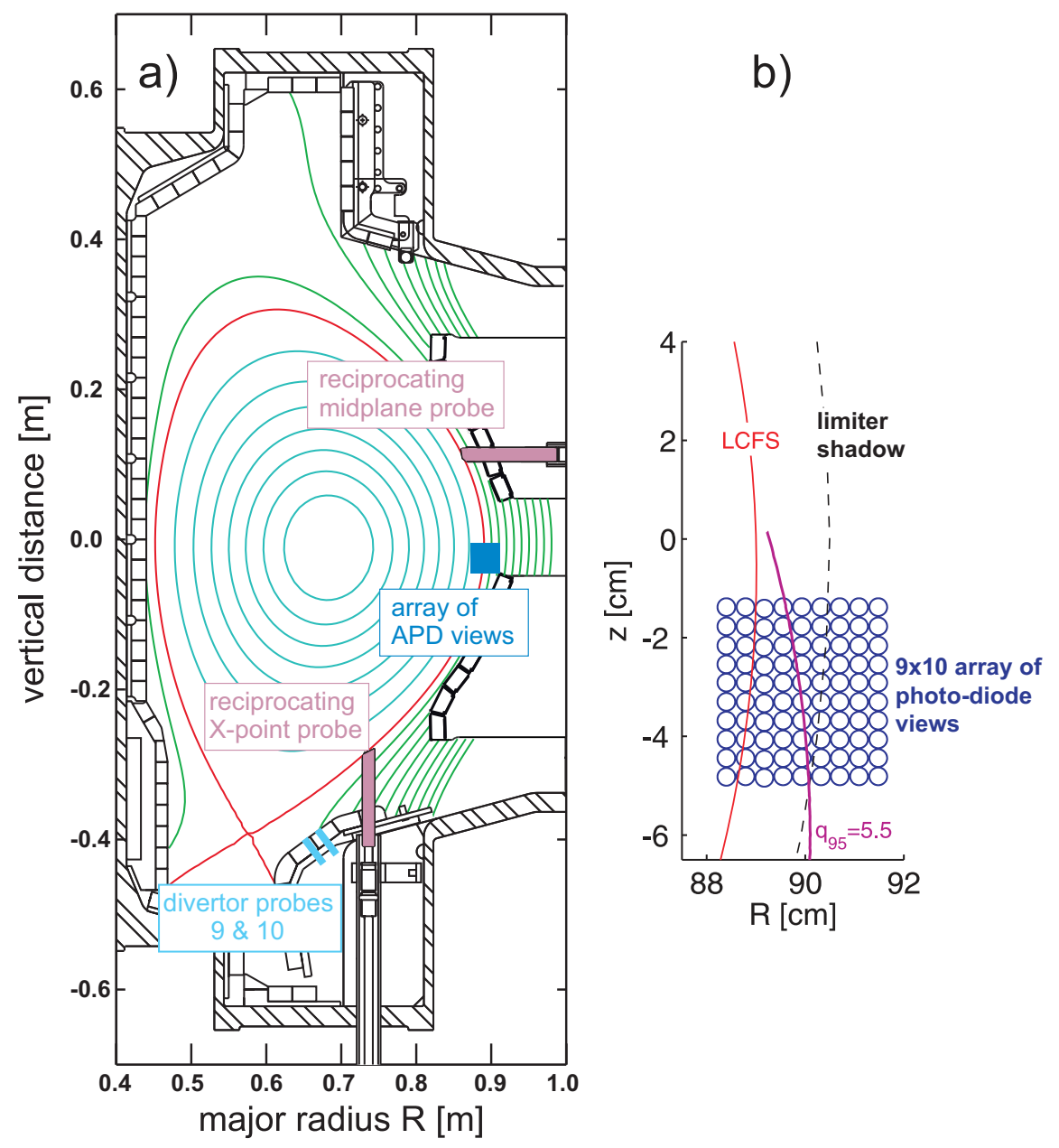

Figure 1. a.) Poloidal cross section of Alcator C-Mod indicating the positions of the probe diagnostics utilized in this work and the f.o.v. of the GPI array of views. b.) Magnetic mapping of the vertical probe plunge (red) into the GPI array f.o.v.

field is $L_{\|}=2.85 \mathrm{~m}$ with a variation over the probe plunge of only $\delta L=8 \mathrm{~cm}$. As depicted in Fig. 2, the discharge parameters for this Configuration are kept essentially constant during the measurements. The probe scans six times through the SOL during each discharge in the indicated time window. The recorded data are segmented into sub-time-series, whose duration corresponds to a certain scanning distance of the probe. A typical distance of $\Delta s=3 \mathrm{~mm}$ is chosen, which corresponds to a time-series length of $\Delta t \approx 1 \mathrm{~ms}$. This value is a compromise between time-series length and spatial resolution. It should be noted that in the correlation evaluation higher spatial resolution results in 


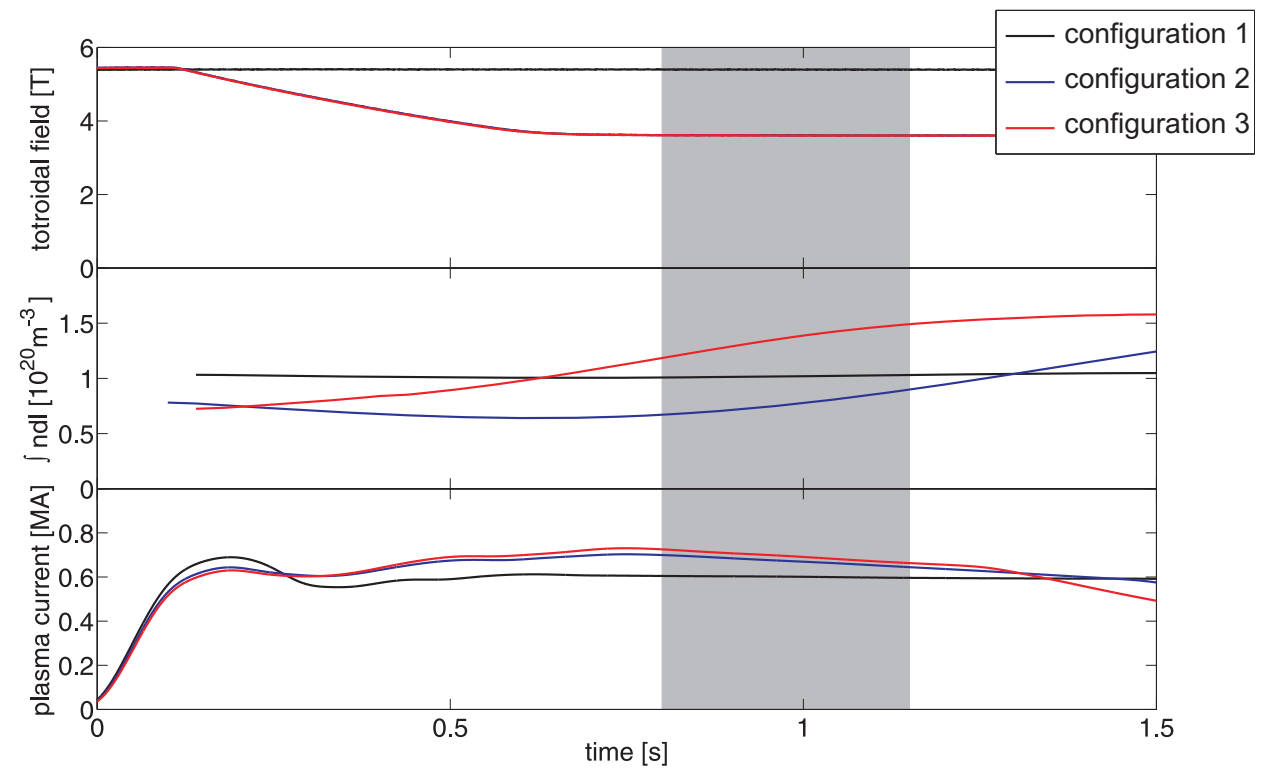

Figure 2. Time traces of the toroidal magnetic field (top), averaged plasma density (middle), and the plasma current (bottom) for the three analyzed configurations. The shaded area indicates the time window during which measurements are taken.

an increase of the maximum correlation amplitude but at the expense of a higher noise level.

Typical radial profiles of the SOL plasma density and the electron temperature are shown in Fig. 3a for this configuration. At the LCFS the plasma density is $n \approx 9 \cdot 10^{19} \mathrm{~m}^{-3}$ at an electron temperature of $T_{e} \approx 70 \mathrm{eV}$. Based on the measured profiles, the characteristic velocities that are believed to play an important role in plasma dynamics parallel to the magnetic field are depicted in Fig. 3b, i.e. the ion sound speed $c_{s}$, Alfvén velocity $v_{A}$, and electron thermal velocity $v_{e, t h e}$. The ion sound speed is in the range 20 to $60 \mathrm{~km} / \mathrm{s}$ and is more than two orders of magnitude smaller than the other two velocities throughout the entire SOL. The electron thermal velocity and Alfvén velocity are of the same order, although the Alfvén velocity is significantly larger in the mid and far SOL with a magnitude of $\approx 2.5 \cdot 10^{4} \mathrm{~km} / \mathrm{s}$ at a distance of $\rho=2 \mathrm{~cm}$ into the SOL.

\subsection{Configurations 2 and 3}

In order to map magnetically between the GPI f.o.v. and divertor-probe 10, $\mathrm{q}_{95}$ was reduced to the range 3.25-3.55 ("Configuration 2" in Table 2). In Configuration 3 divertor-probe 9 was mapped to the GPI view, requiring a q95 of 3.0-3.2. This Configuration 3 mapping was achieved at two different sets of currents and fields in order to investigate dependencies other than $\mathrm{q}_{95}$, as indicated in Table 2. In these configurations the plasma current was decreasing somewhat during the data window, thereby scanning the probe-mapped field-line through the GPI f.o.v. The connection lengths from the GPI f.o.v. to divertor-probes 9 and 10 were similar with $L_{\|}=2.63 \mathrm{~m}$ 

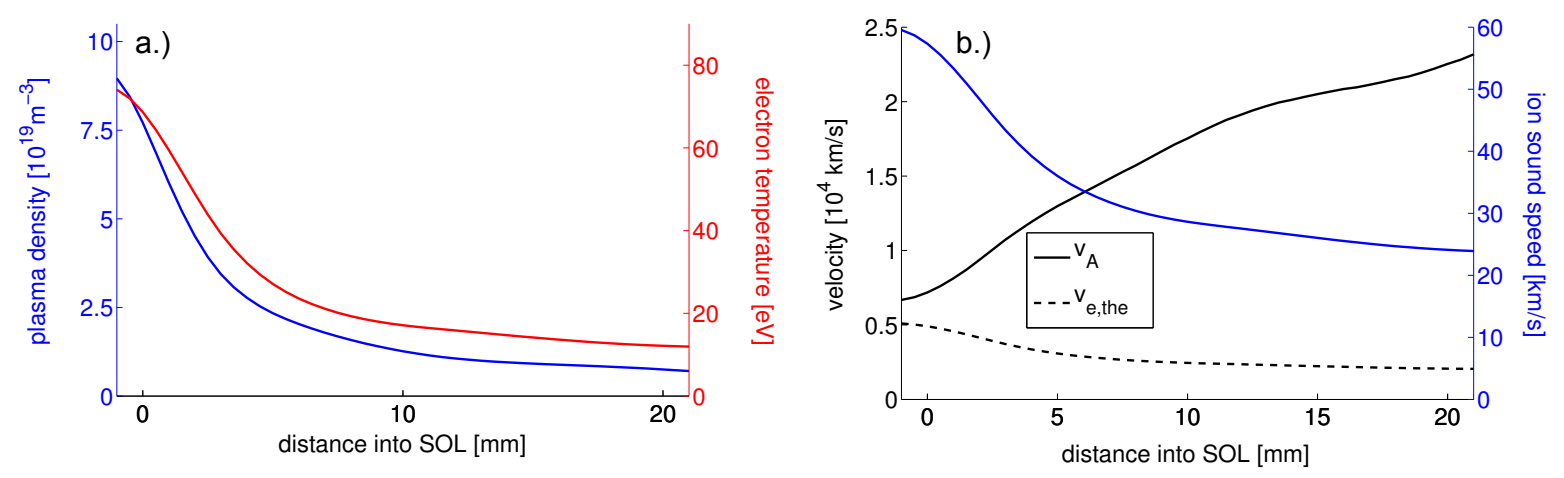

Figure 3. a.) Radial profiles of the plasma density and the electron temperature in the SOL as measured by a reciprocating probe at the outboard midplane, and b.) the resulting radial profile of the electron thermal $v_{e, t h e r m}$, Alfvén $v_{A}$, and ion sound velocity $c_{s}$ (right).

and $L_{\|}=2.64 \mathrm{~m}$, respectively.

\subsection{Synchronization of Timing between Diagnostics}

Because we are investigating the rapid parallel dynamics of the SOL turbulence by cross-correlating time-series signals from two different diagnostics, it is crucial that the timing of the data acquisitions be synchronized at the appropriate level, sub- $\mu$ s in this case. This was accomplished by having each diagnostic's digitizers sample the same TTL "signature" waveform [28], which has multiple fast $(\sim 40 \mathrm{~ns})$ leading and trailing edges. The divertor-probe signals were digitized at $0.5 \mathrm{MS} / \mathrm{s}$, and GPI signals were digitized at $2 \mathrm{MS} / \mathrm{s}$. The edges of the signature waveform are synchronized by aligning those edges that are sampled by the slower digitizer during an edge transition, allowing achievement of a $<0.5 \mu$ s relative timing accuracy between the the two diagnostics. This is demonstrated in Fig. 4 where the probability distribution of time delays at maximum cross-correlation between the two synchronized signature timing waveform signals is plotted. This PDF was constructed from the signature timing signals that were digitized during the discharges of Configuration 2 after synchronizing them (and thus the digitizers' timebases) by post-acquisition processing. The bin size for the PDF is $0.5 \mu \mathrm{s}$, as it is in the analyses of cross-correlations among the SOL fluctuations described in Section 3. This value is taken as the temporal accuracy of the measurements.

\section{Correlation of fluctuations along the magnetic field}

Striking evidence for a strong correlation of fluctuations along the magnetic field is gained even from the raw fluctuation data. Fig. 5 shows an example of simultaneously recorded sub-time-series of the ion saturation current, as measured by the reciprocating probe, and $\mathrm{D}_{\alpha}$ fluctuations, as measured with a single diode out of the two-dimensional view of the GPI array. This particular $\mathrm{D}_{\alpha}$ sub-time-series is from a diode view for which a high cross-correlation with the ion saturation current fluctuations, as measured by the 


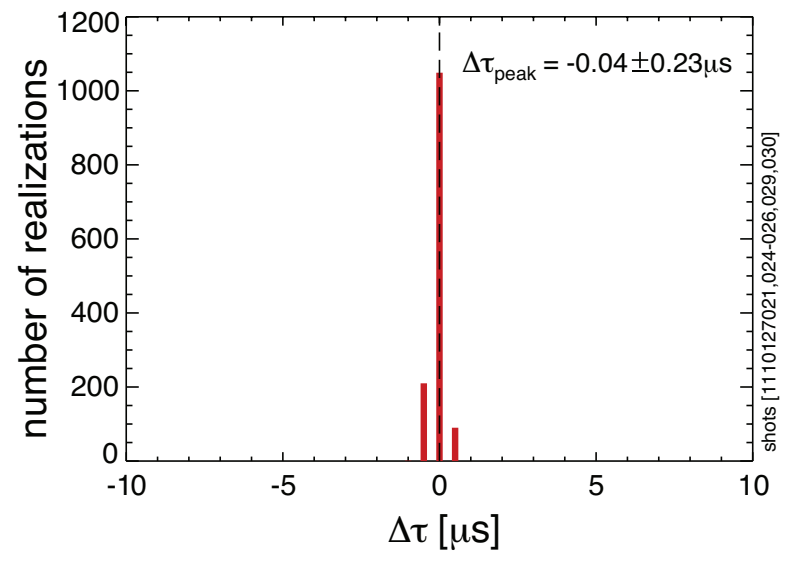

Figure 4. PDF of the time delays at maximum cross-correlation between the timing signals to the GPI and divertor-probe digitizers after timebase synchronization. By design the delays are predominately 0 . The bin size is $0.5 \mu \mathrm{s}$.

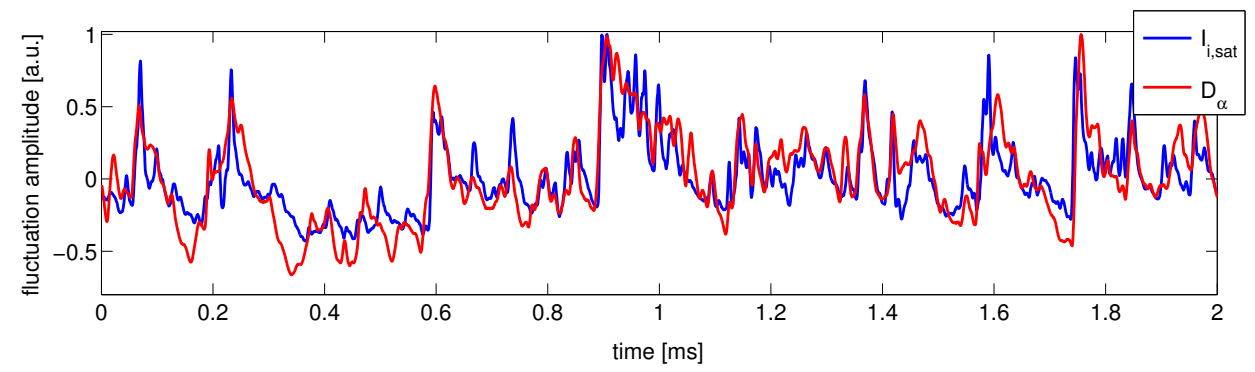

Figure 5. Time-series of the ion saturation current fluctuations $I_{i, s a t}$ as measured by the vertically scanning probe and of $\mathrm{D}_{\alpha}$ fluctuations as measured by a single APD at the outboard midplane.

reciprocating probe, is obtained. It is clearly seen that fluctuation events are observed by both diagnostics. Large-amplitude events especially can be directly identified in both traces, and there is small or zero time delay (to be determined) between the two time-series.

Before cross-correlating GPI emission fluctuations with probe signals, we examine the spatiotemporal structure of fluctuations perpendicular to the field at the location of the 2D array of GPI views, i.e. near the outboard midplane. This is done first by examining the typical auto-correlation functions and cross-correlation functions for emission signals from views near the center of the GPI array. The cross-correlation function is constructed by finding the largest correlation amplitude between any view within the array and the reference view at each time delay value. As seen in Fig. 6a, typical auto-correlation and cross-correlation times are $\approx 20 \mu \mathrm{s}$ and $\approx 25 \mu \mathrm{s}$, respectively. In order to examine the spatial structure, we cross-correlate signals from all $9 \times 10$ views in the GPI array with one near the array center at zero time delay, as shown in Fig. 6b. We typically find an elongated and tilted structure for these events with vertical and radial cross-correlation lengths of $\approx 1.1 \mathrm{~cm}$, consistent with previous $\mathrm{C}$ Mod observations $[5,13]$. To quantify the effect of perpendicular propagation of the 
large structures on the cross-correlation amplitudes we show in Fig. 7 the amplitudes at three different values of time delay, $\tau=(-25,0,25) \mu \mathrm{s}$, during which time the structure is seen to move upward and radially outward. The propagation direction and speed is consistent with previous fast camera recordings of blob motion [29].

a.)

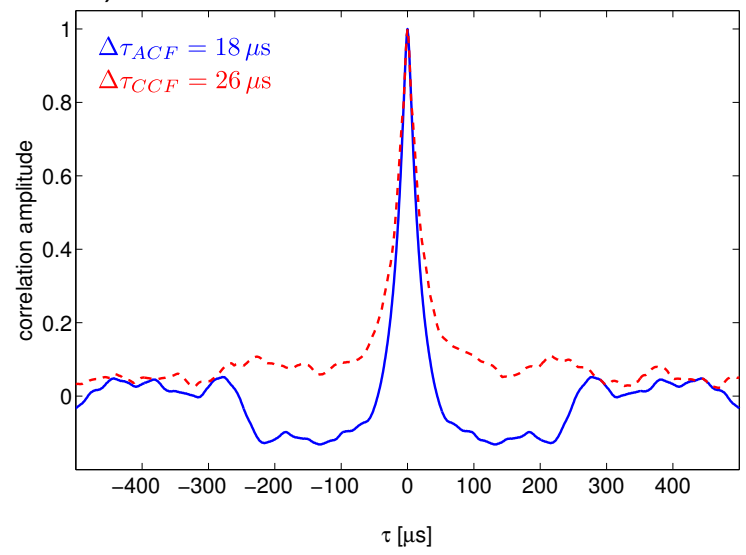

b.)

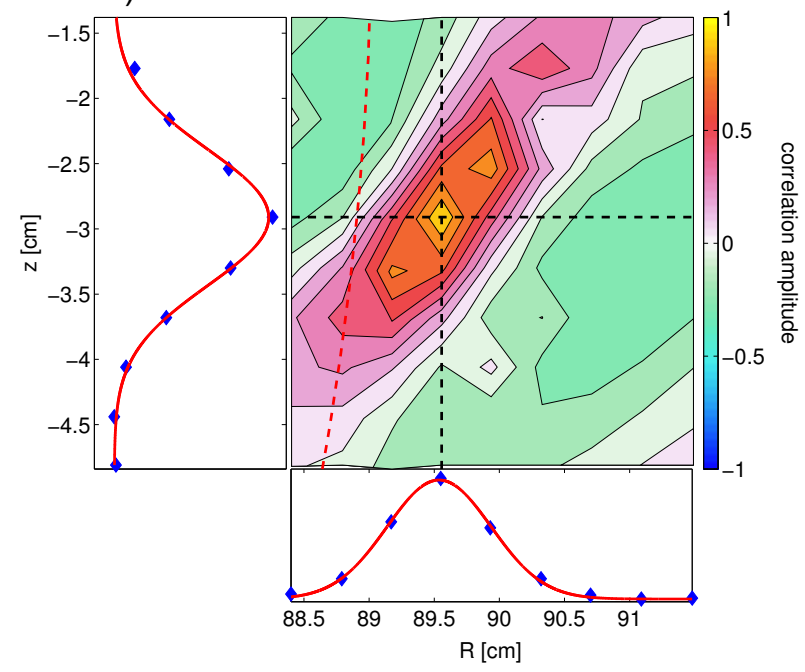

Figure 6. a) Auto-correlation (blue) and cross-correlation function (red) amplitudes at zero time lag $\tau$. b) color-coded cross-correlation amplitudes of one view located at $z=-3 \mathrm{~cm}, R=88.5 \mathrm{~cm}$ with all views in the GPI array at zero time lag. The resulting radial and vertical correlation lengths, as obtained from the Gaussian fit to the respective cuts, are $\delta r=\delta z=1.12 \mathrm{~cm}$. The LCFS is indicated as the red dashed line.
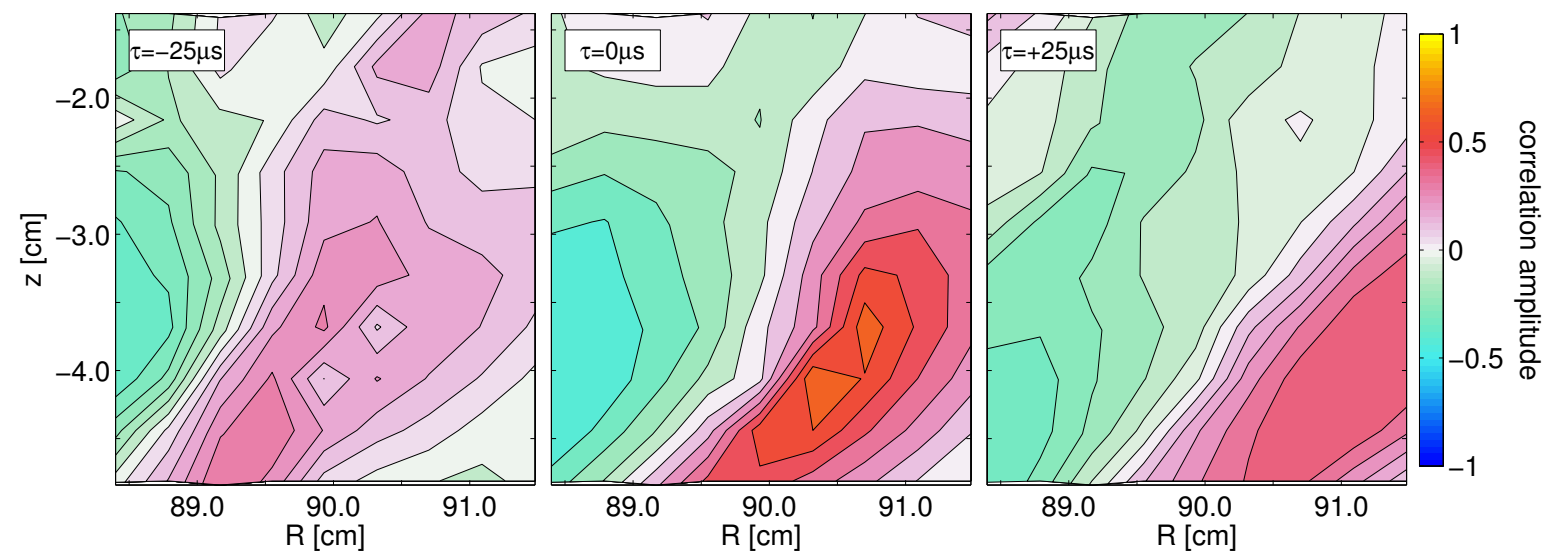

Figure 7. Time evolution of the cross-correlation structure across the GPI f.o.v. over a total time range of $\Delta \tau=50 \mu \mathrm{s}$.

To quantify the correlation apparent in Fig. 5, all 9x10 diode view data are crosscorrelated with the ion saturation current fluctuations for a large number of sub-timeseries. The parallel fluctuation response is then extracted by analyzing the time delay $\Delta \tau$ at which the correlation events are observed. The time delay that yields the maximum 
correlation amplitude for each correlation event is taken as the time it takes for the fluctuations to propagate along the magnetic field. The sign convention used in the analysis is such that negative time delays correspond to events occurring first in the GPI f.o.v. before being observed at the probes.

\subsection{Cross-correlations between Imaging Fluctuations and Scanning Probe Fluctuations}

In this Section we examine the cross-correlations between $\mathrm{D}_{\alpha}$ intensity fluctuations measured by the GPI array and the $I_{i, s a t}$ or floating potential fluctuations measured by the scanning probe. To gain statistical confidence, the analysis is done for ten identical shots with three probe plunges per shot. Sub-time-series are selected corresponding to a probe motion of $\Delta r=3 \mathrm{~mm}$, corresponding to time-series lengths in the range $\Delta t=1-2 \mathrm{~ms}$. The correlation function of each diode and probe sub-time-series is calculated, which yields the spatiotemporal evolution of the correlation across the GPI f.o.v. Events in the spatiotemporal cross-correlation function of each sub-time-series are considered if they fulfill three conditions: (i) the maximum correlation amplitude exceeds a threshold of $60 \%$, (ii) it represents a local maximum in space (i.e. not localized at the edge of the GPI array) and time, and (iii) individual events are temporally separated by a time which is larger than the typical autocorrelation time of $\tau_{\text {auto }}=20 \mu \mathrm{s}$, allowing them to be taken as statistically independent. This way multiple correlation events can be identified within one sub-time-series. A typical map of cross-correlation amplitude between GPI $\mathrm{D}_{\alpha}$ emission and $I_{i, s a t}$ is shown in Fig. 8a. Values over $75 \%$ are found in some cases. The spatial structure resembles very much the GPI correlation structure Fig. 6 with similar poloidal and radial correlation lengths. In Fig. 8b a conditional-averaging analysis has been used to find the spatial structure of density fluctuations $[30,31]$. Large $\mathrm{D}_{\alpha}$ emission events, whose amplitudes exceed $1.5 \sigma$ ( $\sigma$ denotes the standard deviation of emission fluctuations) are taken as the reference signal to obtain the conditional average of either $I_{i, s a t}$ or $\Phi_{f}$ fluctuations measured by the scanning probe. As seen, a dipole potential structure is found to be straddling the maximum in the density structure, consistent with the basic understanding of radial blob propagation dynamics [6] and with previous observations [13, 32]. After constructing the sub-time-series and applying the selection criteria for correlation events, a total number of approximately 5000 correlation events/realizations are collected. A histogram of the observed time delays is depicted in Fig. 9a. Events are observed over the entire considered time-delay range of $\Delta \tau \pm 120 \mu \mathrm{s}$. However, it is clearly observed that the time-delays display a distribution that is peaked close to $\Delta \tau=0$ and is skewed towards negative time delays. Thus, the propagation direction of fluctuations is predominantly from the outboard midplane towards the divertor. This holds not only for the observed skewness of the distribution, but also for its peak, which is found at a time delay of $\Delta \tau=-1.5 \pm 0.5 \mu \mathrm{s}(0.5 \mu \mathrm{s}$ is the accuracy of the timing synchronization $)$, Fig. $9 \mathrm{~b}$. The width of the distribution is rather broad with a standard deviation of $\sigma(\Delta \tau)=3.6 \mu$ s. The shaded regions above the histogrammed time-delays indicate the expected times 

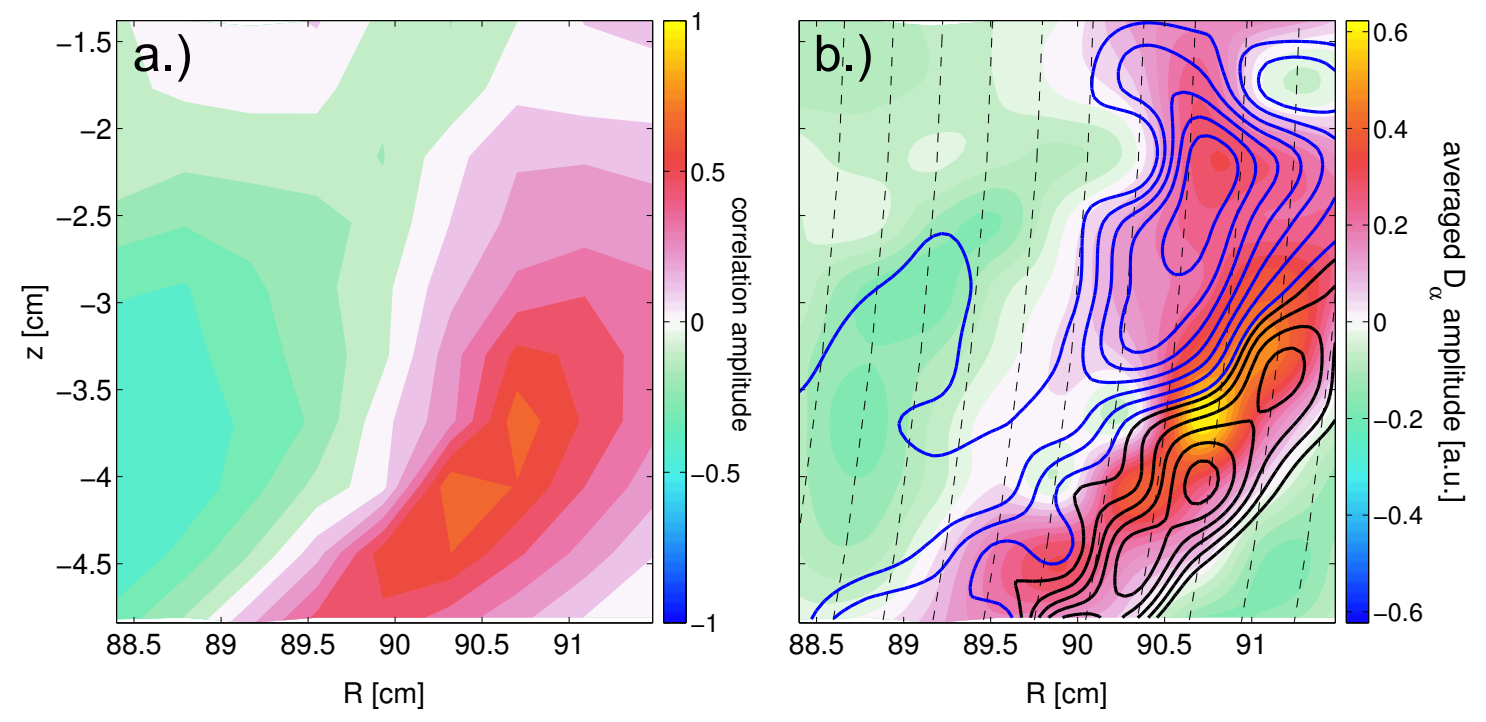

Figure 8. a) Color-coded representation of the correlation amplitude resulting from the cross-correlation analysis between $D_{\alpha}$ and $I_{i, s a t}$ fluctuations. The maximum correlation amplitude is $76 \%$. b) Result of the conditional averaging analysis between $D_{\alpha}$ and $I_{i, s a t}$ fluctuations (color coded) and $\Phi_{f}$ fluctuations (contour lines). Black contour lines represent positive polarity, blue contour lines negative polarity. The dashed lines indicate the flux surfaces.

corresponding to the three different propagation velocities (c.f. Fig. 3) combined with the mean connection length along the magnetic field of $L_{\|}=2.85 \mathrm{~m}$. It is apparent that the time-delay is not given by the ion sound speed (blue region) with a typical time response of $50-100 \mu \mathrm{s}$, consistent with basic dispersion relation considerations (see Section 4). The time-delays expected for a parallel fluctuation velocity given by the Alfvén speed (magenta region in Fig. 9b) and the electron thermal speed (green region) are much smaller. The widths of the shaded regions correspond to the minimum and maximum values through the entire SOL $\rho \leq 2 \mathrm{~cm}$. The peak of the distribution agrees well with a fast parallel response time of fluctuations. The temporal resolution, however, does not allow to clearly distinguish between a propagation at the electron thermal velocity $v_{e, t h e}$ or Alfvén speed $v_{A}$.

\subsection{Cross-correlations between Imaging Fluctuations and Divertor-Probe Fluctuations}

We now examine the emission fluctuations within the imaged region at the outboard midplane and the $I_{i, s a t}$ fluctuations measured by the two divertor-probes. This is done for conditions summarized as Configuration 2 (for mapping to divertor-probe 10) and Configuration 3 (for divertor-probe 9). By connecting with the divertor-probes we extend the investigation of the parallel SOL dynamics beyond the poloidal location of the vertical reciprocating probe to a region that is at the height of the X-point and samples more of the high shear region near it, see Fig. 1. For these two Configurations, He gas was the puffed at the GPI location and He I (587.6 nm) line emission was imaged. As illustrated in Fig. 10, time-series samples of the He I emission and the 

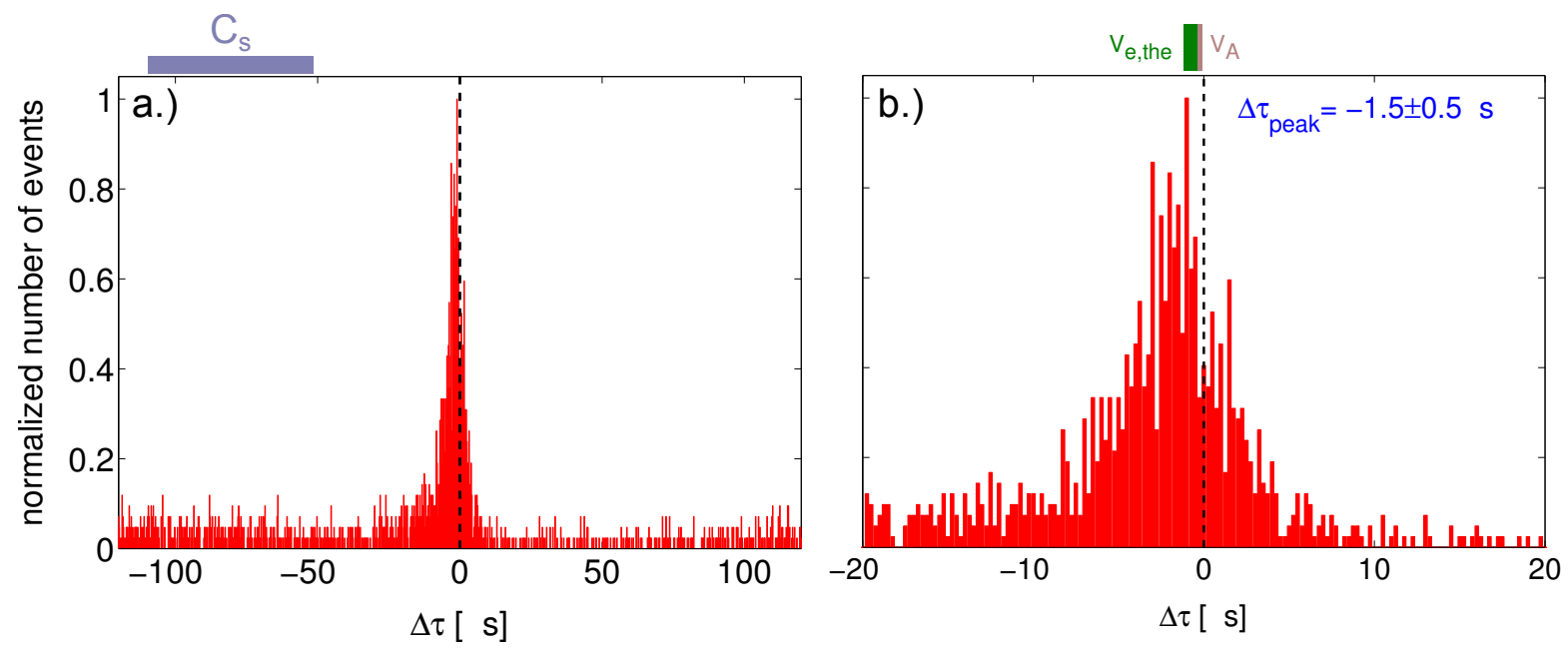

Figure 9. Normalized histogram of the time delay $\Delta \tau$ when the maximum amplitude of individual correlation events is found. The right figure is an enlarged representation around $\Delta \tau=0 \mu \mathrm{s}$. The shaded bars above the graphs indicate the range of time delays resulting from a parallel fluctuation velocity given by the ion sound speed (blue in left figure), Alfvén velocity (magenta in right figure), and electron thermal speed (green).

divertor-probe $I_{i, s a t}$ are also well correlated for the divertor probes (as they were for the reciprocating probe). The spatial structure of the cross-correlation amplitude across the

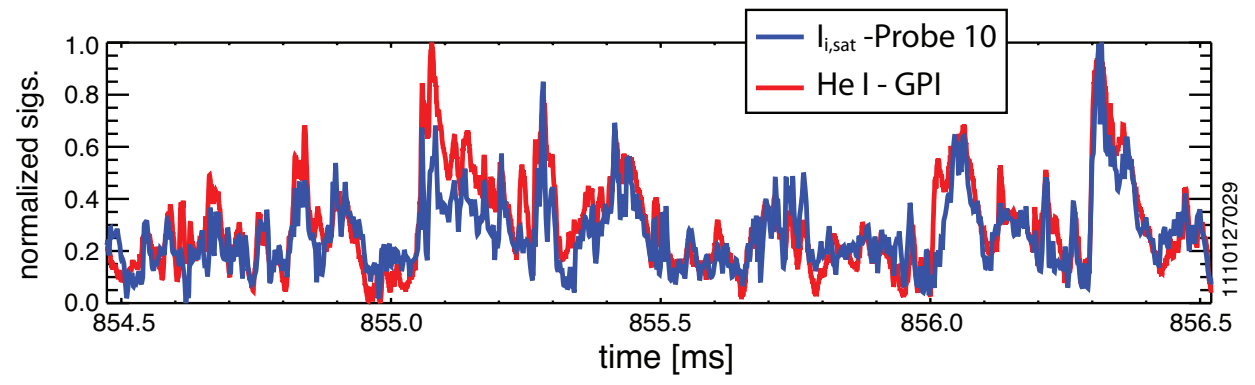

Figure 10. $\mathrm{A} \approx 2$ ms time-series of $I_{i, s a t}$ fluctuations as measured by divertor-probe 10 and of He I emission fluctuations as measured by a single view within the GPI f.o.v. at the outboard midplane. Similarly good correlation between GPI signals and $I_{i, s a t}$ from divertor-probe 9 is found for the magnetic mapping of Configuration 3.

array, shown in Fig. 14 of Section 5, is similar to that of Fig. 8a. For the cross-correlation analysis we chose sub-time-series durations of both $\approx 1 \mathrm{~ms}$ and $\approx 2 \mathrm{~ms}$ and again used the selection criteria that the cross-correlation amplitude for a sub-time-series be larger than a threshold and that the position of the maximum cross-correlation is not on the boundary of the diode view array.

We constructed histograms of the time-delay values that yield the maximum crosscorrelation. The results using the two different durations for the sub-time-series are the same statistically. One of the time-delay histograms (using 616 sub-time-series realizations of length $1 \mathrm{~ms}$ ) is shown in Fig. 11 for Configuration 2 (cross-correlation with divertor-probe 10). We find a mean time-delay of $\Delta \tau=-0.53$ with a standard 
deviation of $\sigma(\Delta \tau)=1.68 \mu \mathrm{s}$ in this case and a mean time-delay of $\Delta \tau=-1.49 \mu \mathrm{s}$, $\sigma(\Delta \tau)=2.26 \mu$ s for Configuration 3 (488 sub-time-series realizations of length $1 \mathrm{~ms}$ cross-correlating with divertor-probe 9). While also here the widths of the distributions are larger than their mean shift, both agree (within error bars) with the GPI-to-scanning probe analysis, yielding a propagation (on-average) from midplane to divertor with a delay time of $\approx 1 \mu \mathrm{s}$, consistent with the previous finding shown in Fig. 9.

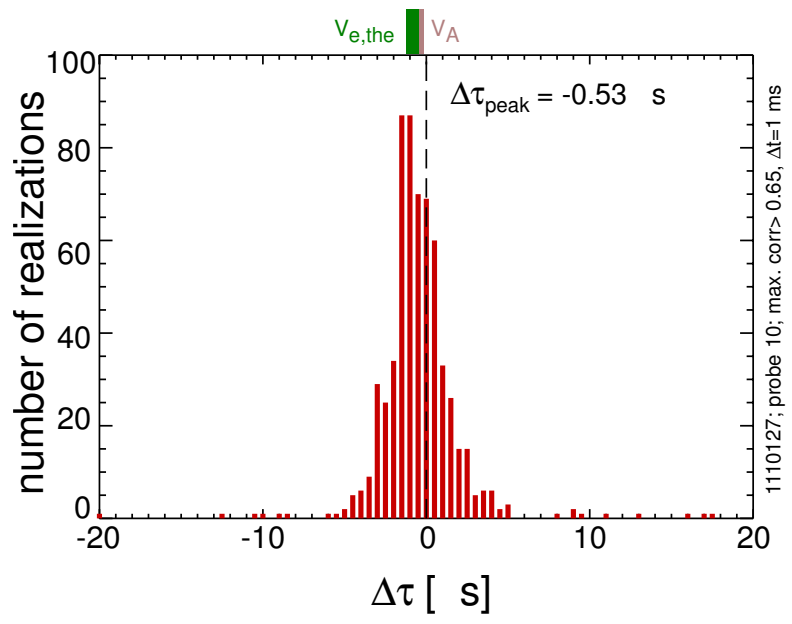

Figure 11. Analogous to Fig. 9, the PDF of time delays, $\Delta \tau$, yielding maximum crosscorrelation between divertor-probe 10 fluctuations and He I emission fluctuations from a point in the GPI f.o.v.. The green shaded bar indicates the time delay resulting from parallel propagation of fluctuations at the electron thermal speed.

\section{Parallel Propagation Velocity of Fluctuations}

The velocity of fluctuations propagating along the magnetic field can be estimated from the parallel electron momentum transport equation expressed by the parallel electron current $J_{\|}$

$$
-\frac{m_{e}}{e} \frac{\partial}{\partial t} J_{\|}=-\nabla_{\|} p+n e \nabla_{\|} \Phi+n e \frac{\partial}{\partial t} A_{\|}-\frac{m_{e} \nu_{e}}{e} J_{\|}
$$

where $p$ denotes the plasma pressure, $m_{e}$ the electron mass, and $e$ the elementary charge. Electron collisions are compiled in the collision frequency $\nu_{e}$. The parallel electric field comprises of the electrostatic and inductive field expressed by the parallel component of the magnetic vector potential $A_{\|}$

$$
E_{\|}=-\nabla_{\|} \Phi-\frac{\partial}{\partial t} A_{\|}
$$

The continuity equation combines the parallel current with the density fluctuation

$$
e \frac{\partial}{\partial t} n=\nabla_{\|} J_{\|}
$$

whereas the perpendicular current is given by the ion polarization drift

$$
\nabla_{\perp} \cdot \mathbf{J}_{\perp}=\frac{n m_{i}}{B^{2}} \frac{\partial}{\partial t}\left(\nabla_{\perp}^{2} \Phi\right)
$$


Using Ampères law and quasineutrality, $\nabla \cdot J=0$ yields the normalized momentum equation

$$
\frac{\partial}{\partial t}(\Psi+\mu J)=C_{s} \nabla_{\|}(N-\Phi)+\mu \nu_{e} J
$$

with normalized parameters

$$
N=\ln n, \quad \Phi=e \Phi / T_{e}, \quad \Psi=A_{\|} / \rho_{s} B, \quad J=J_{\|} / e n C_{s}
$$

( $T_{e}$ electron temperature, $\rho_{s}$ drift scale, $C_{s}$ ion sound speed). Eliminating $J, N$, and $\Phi$ and using the mass ratio $\mu=m_{e} / m_{i}$ and the plasma $\beta\left(=\mu_{0} n T_{e} / B^{2}\right)$ gives a wave equation for the perturbed vector potential

$$
\frac{\partial^{2}}{\partial t^{2}}\left(\beta-\mu \rho_{s}^{2} \nabla_{\perp}^{2}\right) \Psi=C_{s}^{2} \nabla_{\|}^{2}\left(1-\rho_{s}^{2} \nabla_{\perp}^{2}\right) \Psi-\mu \nu_{e} \rho_{s}^{2} \frac{\partial}{\partial t} \nabla_{\perp}^{2} \Psi .
$$

A normal mode analysis of the form

$$
\Psi \sim \exp \{i(k \cdot r-\omega t)\}
$$

yields the dispersion relation. It should be noted that the electron collisions included into the last term of Eq. 5 act as a damping of the wave-like fluctuations and do not contribute to the phase velocity, but can in some cases set the propagation speed as discussed below. The parallel phase velocity $V_{\|}$, given by

$$
\frac{V_{\|}^{2}}{C_{s}^{2}}=\frac{1+\rho_{s}^{2} k_{\perp}^{2}}{\beta+\mu \rho_{s}^{2} k_{\perp}^{2}}
$$

displays two extreme limits: In the kinetic Alfvén wave regime $\beta>\mu$ and the long wavelength limit $\rho_{s}^{2} k_{\perp}^{2} \ll 1$ the parallel phase velocity is given by the Alfvén speed

$$
V_{\|} \approx C_{s} / \sqrt{\beta}=v_{A}
$$

whereas in the inertial Alfvén wave regime $\beta \ll \mu$ and the short wavelength limit $\rho_{s}^{2} k_{\perp}^{2} \gg 1$ the parallel fluctuation velocity is given by the electron thermal speed

$$
V_{\|} \approx C_{s} / \sqrt{\mu}=v_{e, t h e r m} .
$$

Retaining the collisionality term in the latter limit yields for $\nu_{e} \gg \omega$

$$
\omega=i k_{\|}^{2} D_{e}
$$

with $D_{e}=v_{e, t h e r m}^{2} / \nu_{e}$ being an effective diffusion coefficient for the parallel propagation of the potential perturbation. The typical velocity for this parallel diffusion is estimated as

$$
V_{\|, \text {diff }}=k_{\|} D_{e} .
$$

For the SOL parameters of this experiment three cases are obtained when applying this analysis: In the near-SOL $\rho \leq 2 \mathrm{~mm}, k_{\perp}^{2} \rho_{s}^{2} \leq 10^{-2}$, and $\beta / \mu=\mathcal{O}(1)$ a parallel fluctuation velocity of roughly $v_{A}$ is expected. However, in this region the Alfvén and electron thermal speeds are similar, as seen in Fig. 3a. Indeed the the correlation analysis of all three configurations are consistent with these speeds. In the mid- to far- SOL $V_{\|}$approaches the Alfvén speed in the analysis neglecting collisions, and the 
parallel response time is expected to be significantly shorter in this second case. In the far-SOL, i.e. the radial range $\rho \geq 1 \mathrm{~cm}$, we find that collisional damping plays a role

with $\nu_{e} / k_{\|} v_{e . t h e r m} \geq 1$ (where $k_{\|}$is evaluated in Section 5). In this third case the parallel diffusion speed $V_{\|}$,diff is close to or greater than the electron thermal velocity, which is also consistent with the experimental finding. We note that in contrast to the Alfvén and electron thermal speeds, the diffusion velocity is much more sensitive to variations of the plasma density and temperature, mainly through $\nu_{e}$. Because these quantities vary between realizations of the correlation events due to fluctuations, significant variations in propagation times (as is seen experimentally) are expected if this is the dominant mechanism.

\section{Estimate of parallel wavelength}

If the position of the peak correlation on the array is compared to the puncture point for the field line from the probe, as calculated based on the EFIT equilibrium, a systematic deviation is observed. An example is shown in Fig. 12. The calculated puncture point of the probe-position field line with the diode array f.o.v., indicated as the red square, is displaced from the region of maximum correlation by $\Delta_{\theta} \approx 1.3 \mathrm{~cm}$. However, the displacement is only in the poloidal direction along the flux surface and the maximum correlation is found when the probe-position field line intersects with that flux surface, shown as the solid black line in Fig. 12. Several effects could result in this displacement: An error in the equilibrium calculation would give an inaccurate mapping calculation. The required error in the magnetic field, however, would need to be $\approx 5 \%$ which is much larger than the accuracy with which the toroidal field and plasma current are measured [33].

The radial accuracy of the EFIT reconstruction at the outboard midplane is typically $\delta r \leq 3 \mathrm{~mm}$. Due to magnetic shear this uncertainty results in a possible poloidal displacement of $\Delta_{\theta} \leq 1 \mathrm{~cm}$ in regions close to the X-point. However, this possible displacement is also less than what is observed. Another effect is the propagation of the fluctuation filament since the maximum correlation is observed with a non-zero time delay. Radial and poloidal propagation velocities of large amplitude blobs in the SOL at the outboard midplane are comparable with $v_{r} \approx v_{p} \approx 0.5-1 \mathrm{~km} / \mathrm{s}$ $[34,13]$, leading to an upper limit for the displacement of $\Delta_{\theta} \approx 1-2 \mathrm{~mm}$ during the typically observed parallel propagation time. This is also much smaller than the displacement observed in the present case. There are also relatively strong toroidal flows in the SOL, with typical flow velocities of $v_{t} \approx 10 \mathrm{~km} / \mathrm{s}$ for low-density lower single null discharges [35] that might lead to such a displacement. At the position of the probe close to the lower $\mathrm{X}$-point the pitch angle of the magnetic field is small with $\xi \approx 8^{\circ}$. Thus, the maximum vertical motion of the intersection point of the filament with the poloidal plane during the propagation time is also small with $\Delta_{\theta} \approx 2-4 \mathrm{~mm}$ and cannot account for the observed displacement either. As a result, we ascribe the observed displacement to the existence of a non-zero wavelength of the fluctuations parallel to the magnetic 


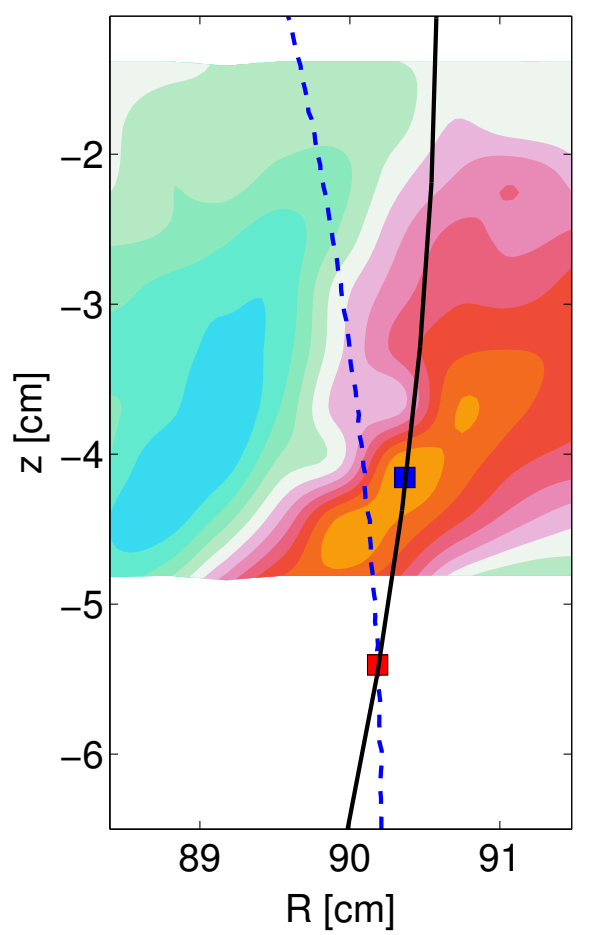

Figure 12. Color-coded representation of the cross-correlation amplitude between $I_{i, s a t}$ and $\mathrm{D}_{\alpha}$ fluctuations across the GPI f.o.v. together with the result of the field line mapping calculation of the probe plunge (dashed blue) and one flux surface (solid black). The peak of the correlation amplitude is marked as the blue square, while the red square marks the puncture point of the fieldline from the actual position of the probe at the time instant when the maximum correlation is found.

field. From the poloidal separation between the mapping calculations and the position of the maximum correlation, $\Delta_{\theta}$, an average parallel wavenumber $\left\langle k_{\|}\right\rangle$is estimated [36] from

$$
\left\langle k_{\|}\right\rangle=\left\langle k_{\theta}\right\rangle \tan \alpha
$$

where $\alpha$ is the angle between the wavenumber $k$ and its poloidal component $k_{\theta}$ and is estimated asgunn

$$
\tan \alpha=\Delta_{\theta} / L_{\|} .
$$

A characteristic poloidal wavenumber for the blobs is obtained from analysis of the nearmidplane imaging. As seen in Fig. 13 a spectral peak exists around $k_{\theta}=(4.3 \pm 1.3) \mathrm{cm}^{-1}$, with the variation estimated from its $1 / e$-folding width. The poloidal distance between probe mapping and maximum correlation, including the maximum variation due to poloidal structure propagation and toroidal plasma flows, is $\Delta_{\theta}=(1.3 \pm 0.6) \mathrm{cm}$. The connection length parallel to the magnetic field including the maximum variation across the GPI f.o.v. is $L_{\|}=(285 \pm 4) \mathrm{cm}$. The resulting mean parallel wavenumber even within the limits of the maximum confidence interval is non-zero with $\left\langle k_{\|}\right\rangle=$ $(2.9 \pm 1) \cdot 10^{-2} \mathrm{~cm}^{-1}$. To compare this value to the parallel wavenumber expected for resistive ballooning modes $k_{\|, R B}$ we assume different limits for $k_{\|, R B}$ : The parallel scale 
length of those modes is typically taken as the field line connection length $L_{\|}=2 \pi q R$, however, for strongly ballooning modes only the outboard length $L_{\|}=\pi q R$ seems more appropriate. Additionally, instead of the edge value $q_{95}=5.5$ the local outboard midplane safety factor $q_{S O L}=1.9$ can be used. The different estimates yield a range of the expected wavenumber $k_{\|, R B}=2 \pi / L_{\|} \approx(0.2 \ldots 1) \cdot 10^{-3} \mathrm{~cm}^{-1}$, which is smaller than what we obtain in the measurements.

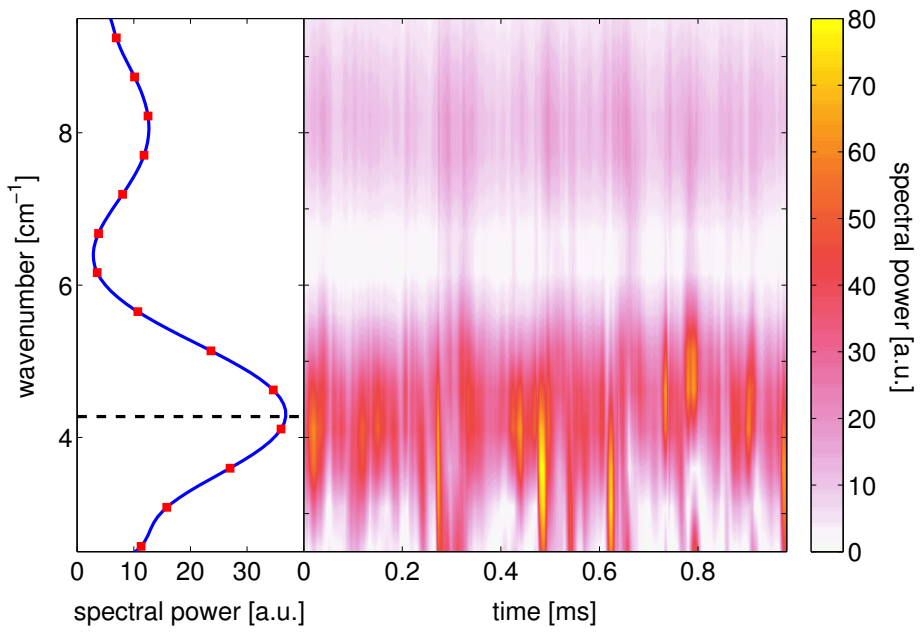

Figure 13. Color-coded plot of the time evolution of the poloidal wavenumber spectrum and time-averaged poloidal wavenumber spectrum indicating the peak at $k_{p o l}=4.3 \mathrm{~cm}$.

A similar analysis has been done for results from Configurations 2 and 3, where the divertor-probes mapped to the GPI views. A similar observation is obtained, i.e. that the EFIT-mapped field line from the divertor-probe punctures the plane of GPI f.o.v. and is not found at the point of maximum cross-correlation amplitude, but at a point displaced vertically below and slightly outside it. This is illustrated in Fig. 14, where a contour of cross-correlation amplitudes is shown along with the puncture point for the divertor-probe field line. As is evident in Fig. 14, most of the magnitude of $\Delta_{\theta} \approx 3.2 \mathrm{~cm}$ comes from the vertical displacement between the cross-correlation maximum and the probe-field-line puncture point. A histogram for the vertical-displacements is shown in Fig. 15. Also shown in Fig. 15 is a comparison between the vertical-displacements for shots with the same $q_{95}$, but with different values of toroidal field and plasma current. Since the vertical-displacements are found to be quite similar, we conclude that the displacement does not depend strongly on the separate magnitudes of field and current. As shown in Fig. 15, for Configuration 3 the mean vertical-displacement is $\Delta_{\theta}=3.7 \pm 0.5 \mathrm{~cm}$, while the mean value for $\Delta_{\theta}$ in the Configuration 2 case is $2.9 \pm 0.3 \mathrm{~cm}$. Since plasma currents are decreasing slightly in both of these configurations, the point of maximum cross-correlation moves over the full vertical extent of the f.o.v., and that motion correlates very well to motion of the EFIT-mapped puncture point of the field-line from the probe. The cross-correlation between the motions is $93 \%$, indicating that filament-mapping follows quite closely relative changes in the field-line 


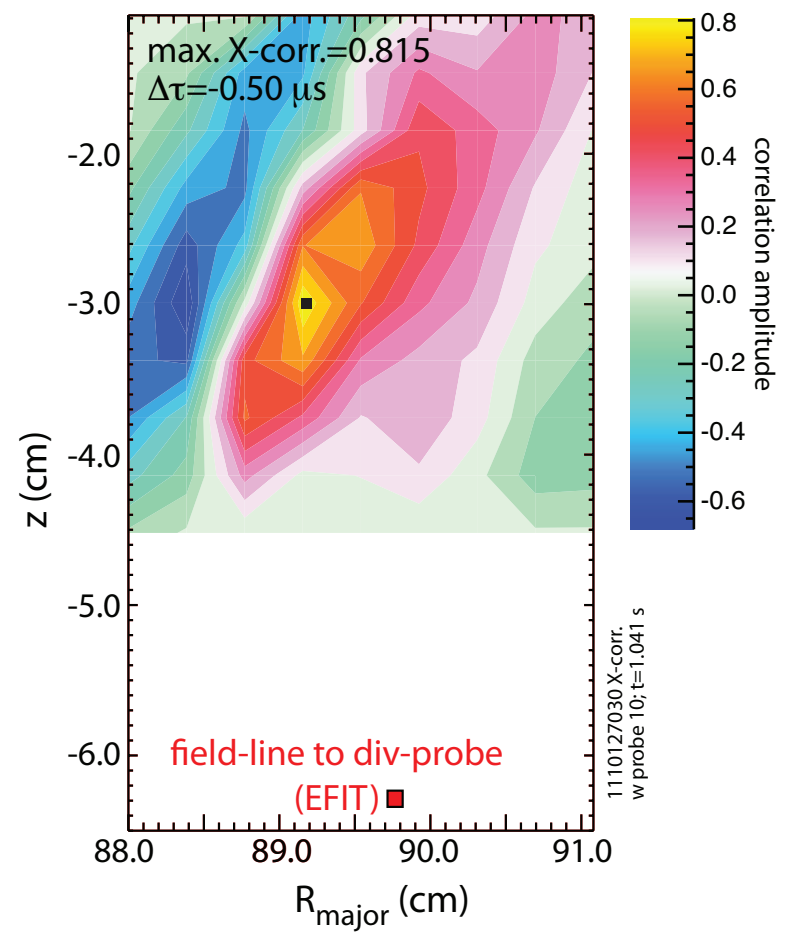

Figure 14. Analogous to Fig. 12, the spatial dependence of cross-correlation amplitudes between $I_{i, s a t}$ from divertor-probe 10 and the He I emission across the GPI f.o.v. The time delay to produce the maximum cross-correlation was $-0.5 \mu \mathrm{s}$ (emission fluctuation leading the $I_{i, s a t}$ fluctuation) for this $2 \mathrm{~ms}$ sub-time-series. The peak of the correlation amplitude (81\%) is marked with the black square symbol. Also shown is the EFIT-mapped puncture point of the field line from divertor-probe 10 (red square).

mapping, albeit with the noted displacement. As with Configuration 1, we attribute the displacement to a non-zero parallel wavelength of the fluctuation. Analyses of the imaging data for the shots of Configurations 2 and 3 yield $k_{\theta} \approx 2.7 \mathrm{~cm}^{-1}$ for filaments at the outboard midplane. Thus, using the measured mean displacement between the location of the maximum cross-correlation and the probe field-line puncture point and a mean connection length $\mathrm{L}_{\|}=2.64 \mathrm{~m}$, the implied parallel wavenumber is $\left\langle k_{\|}\right\rangle=(3.0 \pm 1) \cdot 10^{-2} \mathrm{~cm}^{-1}$ for Configuration 2 and $\left\langle k_{\|}\right\rangle=(3.8 \pm 1.3) \cdot 10^{-2} \mathrm{~cm}^{-1}$ for Configuration 3 , both of which are comparable to, but slightly larger than the value obtained in Configuration 1. It is also larger than expected for resistive ballooning modes. 


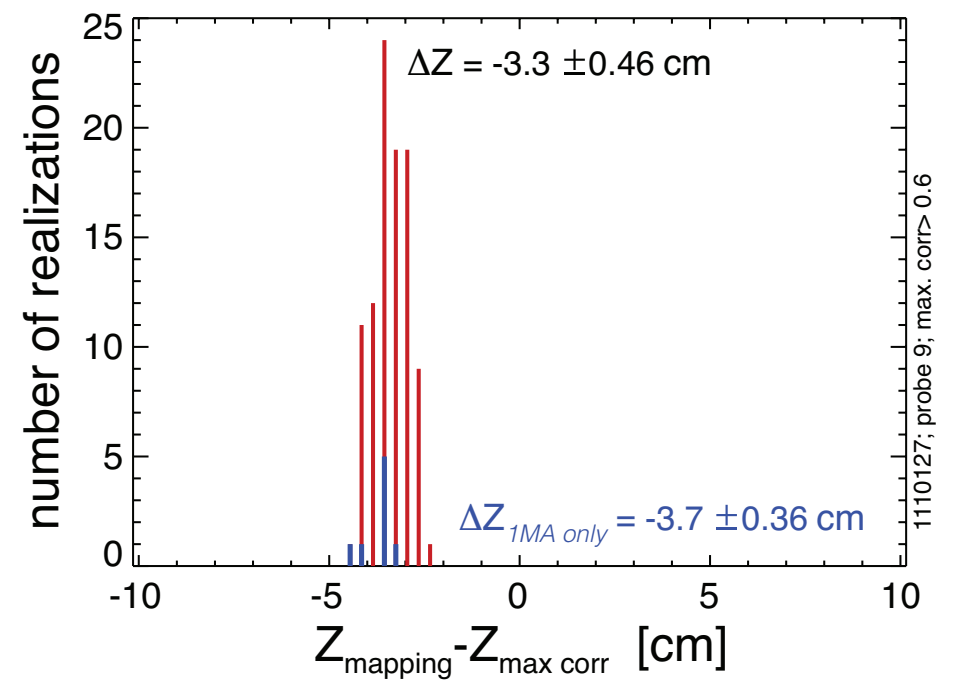

Figure 15. Histogram of the vertical displacement between the puncture point for the field line from divertor-probe 9 and the point of maximum $I_{i, \text { sat }}$-to-He I crosscorrelation within the GPI array f.o.v. Even though the mapping was being scanning vertically through the f.o.v. (by decreasing $\mathrm{I}_{p}$ ), there remains a tight cluster around $\Delta_{\theta}=-3.7 \pm 0.5 \mathrm{~cm}$, indicating that the two points track together. In blue are the histogrammed distances for those cases in Configuration 3 at higher current and field $\left(I_{P} \approx 1 \mathrm{MA} \& B=4.6 \mathrm{~T}\right.$ vs $\left.I_{P} \approx 0.78 \mathrm{MA} \& B=3.6 \mathrm{~T}\right)$. The sub-time-series durations are $2 \mathrm{~ms}$ for these results. 


\section{Summary and Discussion}

We have investigated the parallel dynamics of blob/filaments in the Alcator C-Mod SOL by examining the cross-correlations between fluctuation-sensitive diagnostics that are toroidally and poloidally displaced from each other, but are approximately connected magnetically. With one of the diagnostics at the outboard midplane region and the others near the X-point region or at the divertor target, we have found that filament fluctuations do extend from midplane to outer target, at least for SOL filaments with $\rho$ at the target greater than $0.85 \mathrm{~cm}$. SOL filaments are observed to connect between the outboard midplane and the location of the scanning probe with connection lengths of $L_{\|} \approx 2.8 \mathrm{~m}$. Generally, strong magnetic shear can de-correlate fluctuation filaments due to an increase of the cross-field polarization currents [16, 37]. However, for the present experimental configurations $k_{\theta}^{2} \rho_{s}^{2} \ll 1$ holds even in the X-point region [17], and this effect is not expected to influence significantly the parallel filament structure, consistent with the observed high values of correlation. The 2D spatial coverage of the GPI allows determination of the spatial maximum in the cross-correlation amplitude between fluctuations measured within its f.o.v. and elsewhere. Knowing the spatial maximum then allows a quantitative measurement of the delay time between fluctuation events, thereby determining the propagation direction and an estimate of the parallel propagation velocity. We find that the sense of propagation in bottom low-fieldside region of the plasma is on-average more away from midplane toward the lower divertor targets. The mean delay time between events correlated in the diagnostics' signals $(\approx 1 \mu \mathrm{s})$ is consistent with fast parallel propagation velocities of the filament fluctuations at the electron thermal and Alfvén velocity. This result is in contrast to similar previous measurements [13] in which a time delay for maximum correlation of typically $\Delta \tau=20 \mu \mathrm{s}$ was observed, considerably larger than the $\approx 1 \mu \mathrm{s}$ found in the present study. Furthermore, the observed maximum correlation amplitudes in this study are much larger than the $C_{\max } \approx 30 \%$ observed previously. Both of these differences are most likely due to the fact that previously the GPI spatial coverage was a single radial row of views crossed by a single vertical column of views. Thus the location of the cross-correlation maximum was probably not being observed; instead it was assumed to be the EFIT-mapped probe location, which in the present study has been shown to be displaced from the position of maximum correlation by $\Delta_{\theta} \geq 1 \mathrm{~cm}$, c.f. Figs. 12 and 14. Thus the larger time delay was not predominantly determined by the parallel propagation of fluctuations, but mostly by the time it takes for the blob to propagate into the singular diode view, since the characteristic poloidal blob velocity for the studied discharges is $v_{p} \approx 500 \mathrm{~m} / \mathrm{s}$, yielding the previously observed time delay of $\Delta \tau=\Delta r / v_{p}=20 \mu \mathrm{s}$. This finding demonstrates the importance of spatiotemporal diagnostics to localize the position of the filament to avoid any contribution of perpendicular propagation. In the mid- and far-SOL (where a majority of correlation events were been measured), a fast parallel response time corresponding to a velocity that is close to or greater than the electron thermal velocity is not only expected 
from wave propagation, but also from diffusion of the potential along the magnetic field. Thus, diffusion is also a possible explanation of the observed peak in the response time distribution functions. This conclusion, however, is based on the assumption that fluctuations are generated close to the outboard midplane propagate/diffuse along the magnetic field towards the divertor. Based on SOL measurements in Tore Supra it has been observed that enhanced radial transport is significantly spread poloidally around the outboard midplane [38] suggesting that the generation of turbulent structures is not well localized at the midplane. This could explain the widths of the response time distribution functions and even the observation of positive time lags, i.e propagation towards the outboard midplane, since both depend on the actual localization of the generation and its variation. Additionally, plasma parameter fluctuations affect the response time, particularly for a diffusive response, which is much more sensitive to plasma density and temperature when compared to the wave phase velocity limits. It should be noted that from the correlation analysis no evidence is found for a significantly slower parallel response time of fluctuation filaments, particularly on the order of the ion sound speed. This finding stands in contrast to numerical simulation results, in which a subsonic parallel particle transport is observed [39].

Finally, we find that, while the filaments map approximately along the field lines, the mapping is not exactly field-aligned, with a deviation that is outside the expected accuracy of the EFIT equilibrium reconstruction or propagation distances within the observed parallel propagation times. We interpret the deviation of the filament from the field line to a non-zero parallel wavenumber, and estimate the magnitude in the range $\left\langle k_{\|}\right\rangle \approx(3-4) \cdot 10^{-2} \mathrm{~cm}^{-1}$, which is larger than expected from resistive ballooning.

\section{Acknowledgments}

This work is supported by US DoE Coop. Agreement DE-FC02-99ER54512.

\section{References}

[1] D’Angelo N. Ion Waves in an Inhomogeneous Plasma. Phys Fluids. 1963;6:592-593.

[2] Chu TK, Hendel HW, Politzer PA. Measurements of enhanced plasma losses caused by collisional drift waves. Phys Rev Lett. 1967;19(19):1110-1113.

[3] Horton W. Drift waves and transport. Rev Mod Phys. 1999;71(3):735-778.

[4] Terry JL, Maqueda R, Pitcher CS, Zweben SJ, LaBombard B, Marmar ES, et al. Visible imaging of turbulence in the SOL of the Alcator C-Mod tokamak. J Nucl Mater. 2001;290-293:757-762.

[5] Zweben SJ, Stotler DP, Terry JL, LaBombard B, Greenwald M, Muterspaugh M, et al. Edge turbulence imaging in the Alcator C-Mod tokamak. Phys Plasmas. 2002;9(5):1981-1989.

[6] D'Ippolito DA, Myra JR, Zweben SJ. Convective transport by intermittent blob-filaments: Comparison of theory and experiment. Phys Plasmas. 2011;18:060501-1-060501-48.

[7] Katz N, Egedal J, Fox W, Le A, Porkolab M. Experiments on the propagation of plasma filaments. Phys Rev Lett. 2008 Jul;101(1):015003.

[8] Furno I, Spolaore M, Theiler C, Vianello N, Cavazzana R, Fasoli A. Direct Two-Dimensional Measurements of the Field-Aligned Current Associated with Plasma Blobs. Phys Rev Lett. 2011;106:245001-1 - 245001-4. 
[9] Windisch T, Grulke O, Klinger T. Radial propagation of structures in drift wave turbulence. Phys Plasmas. 2006 Dec;13(12):122303.

[10] Carter T. Intermittent turbulence and turbulent structures in a linear magnetized plasma. Phys Plasmas. 2006;13:010701-1 - 010701-4.

[11] Myra JR, Russell DA, D'lppolito DA. Transport of perpendicular edge momentum by driftinterchange turbulence and blobs. Phys Plasmas. 2008 Mar;15(3):032304.

[12] Xu GS, Naulin V, Fundamenski W, Hidalgo C, Alonso JA, Silva C, et al. Blob/hole formation and zonal-flow generation in the edge plasma of the JET tokamak. Nucl Fusion. 2009 Sep;49(9):092002.

[13] Grulke O, Terry JL, LaBombard B, Zweben SJ. Radially propagating fluctuation structures in the scrape-off layer of Alcator C-Mod. Phys Plasmas. 2006;13:012306-1 - 012306-7.

[14] Garcia OE, Naulin V, Nielsen AH, Rasmussen JJ. Computations of intermittent transport in scrape-off layer plasmas. Phys Rev Lett. 2004;92(16):165003-1 - 165003-4.

[15] Garcia OE, Horacek J, Pitts RA, Nielsen AH, Fundamenski W, Graves JP, et al. Interchange turbulence in the TCV scrape-off layer. Plasma Phys Controlled Fusion. 2006 Jan;48(1):L1L10.

[16] Myra JR, Russell DA, D'Ippolito DA. Collisionality and magnetic geometry effects on tokamak edge turbulent transport. I. A two-region model with application to blobs. Phys Plasmas. 2006 Nov;13(11):112502.

[17] Terry JL, Zweben SJ, Umansky MV, Cziegler I, Grulke O, LaBombard B, et al. Spatial structure of scrape-off-layer filaments near the midplane and X-point regions of Alcator-C-Mod. J Nucl Mater. 2009 Jun;390-91:339-342.

[18] Zweben SJ, Terry JL, Agostini M, Davis WM, Diallo A, Ellis RA, et al. Comparison of edge turbulence imaging at two different poloidal locations in the scrape-off layer of Alcator C-Mod. Phys Plasmas. 2013;20:072503-1-072503-18.

[19] Xu XQ, Cohen RH, Porter GD, Rognlien TD, Ryutov DD, Myra JR, et al. Turbulence studies in tokamak boundary plasmas with realistic divertor geometry. Nuclear Fusion. 2000 Mar;40(3Y):731-736.

[20] Umansky MV, Rognlien TD, Xu XQ, Cohen RH, Nevins WM. Turbulence in the divertor region of tokamak edge plasma. Contributions To Plasma Physics. 2004;44(1-3):182-187.

[21] Thomsen H, Endler M, Bleuel J, Chankin AV, Erents SK, Matthews GF, et al. Parallel correlation measurements in the scrape-off layer of the Joint European Torus. Phys Plasmas. 2002;9(4):1233-1240.

[22] Rudyj A, Bengtson RD, Carlson A, Giannone L, mer MK, Niedermeyer H, et al. In: Segre S, Knoepfel H, Sindoni E, editors. 16th EPS Conference on Controlled Fusion and Plasma Physics, Venice; 1989. .

[23] Maqueda1 RJ, Stotler DP, the NSTX Team. Intermittent divertor filaments in the National Spherical Torus Experiment and their relation to midplane blobs. Nucl Fusion. 2010;50:0750021-075002-12.

[24] Zweben SJ, Medley SS. Visible imaging of edge fluctuations in the TFTR tokamak. Phys Fluids B. 1989;1(10):2058-2065.

[25] Bleuel J, Endler M, Niedermeyer H, Schubert M, Thomsen H, the W7-AS team. The spatial structure of edge fluctuations in the Wendelstein 7-AS stellarator. New J Phys. 2002;4:38.138.38 .

[26] Terry JL, Zweben SJ, Bose B, Grulke O, Marmar ES, Lowrance J, et al. High speed movies of turbulence in Alcator C-Mod. Rev Sci Instrum. 2004;75(10):4196-4199.

[27] Cziegler I, Terry JL, Hughes JW, LaBombard B. Experimental studies of edge turbulence and connement in Alcator C-Mod. Phys Plasmas. 2010;17:056120-1 - 056120-8.

[28] Stillerman J, Burke W, Labombard B. An optical timing verification system for Alcator C-Mod. Fusion Engineering and Design. 2010 Jul;85(3-4):367-369.

[29] Grulke O, Terry JL, Zweben SJ. Scaling of radial turbulent structure velocities in the tokamak 
Experimental investigation of the parallel structure of fluctuations in the scrape-off layer of Alcator C-Mod 2:

SOL. In: Schittenhelm M, Bartiromo R, Wagner F, editors. 33rd EPS Conference on Controlled Fusion and Plasma Physics (Rome). vol. 21A, part IV. European Physical Society; 2006. p. 16131616.

[30] Johnsen H, Pécseli HL, Trulsen J. Conditional eddies in plasma turbulence. Phys Fluids. 1987;30(7):2239-2254.

[31] Boedo JA, Rudakov D, Moyer R, Krasheninnikov S, Whyte D, McKee G, et al. Transport by intermittent convection in the boundary of the DIII-D tokamak. Phys Plasmas. 2001;8(11):48264833.

[32] Furno I, Theiler C, Lancon D, Fasoli A, Iraji D, Ricci P, et al. Blob current structures in TORPEX plasmas: experimental measurements and numerical simulations. Plasma Phys Control Fusion. 2011 Dec;53(12):124016.

[33] Granetz RS, Hutchinson IH, Gerolamo J, Pina W, Tsui C. Magnetic diagnostics in Alcator CMOD. Rev Sci Instrum. 1990;61:2967 - 2969.

[34] Terry JL, Zweben SJ, Grulke O, Greenwald MJ, LaBombard B. Velocity fields of edge/ScrapeOff-Layer turbulence in Alcator C-Mod. J Nucl Mater. 2005;337-339:322-326.

[35] LaBombard B, Rice JE, Hubbard AE, Hughes JW, Greenwald M, Irby J, et al. Transport-driven Scrape-Off-Layer flows and the boundary conditions imposed at the magnetic separatrix in a tokamak plasma. Nucl Fusion. 2004;44:1047-1066.

[36] Ritz CP, Powers EJ, Rhodes TL, Bengtson RD, Gentle KW, Lin H, et al. Advanced plasma fluctuation analysis techniques and their impact on fusion research. Rev Sci Instrum. 1988;59(8):1739-1744.

[37] Helander P, Beidler CD, Bird TM, Drevlak M, Feng Y, Hatzky H, et al. Stellarator and tokamak plasmas: a comparison. Plasma Phys Control Fusion. 2012;54(124009):1-12.

[38] Gunn JP, Boucher C, Dionne M, Duran I, Fuchs V, Loarer T, et al. Evidence for a poloidally localized enhancement of radial transport in the scrape-off layer of the Tore Supra tokamak. J Nucl Mater. 2007 Jun;363:484-490.

[39] Havlickova E, Fundamenski W, Naulin V, Nielsen AH, Zagorski R, Seidl J, et al. Steady-state and time-dependent modelling of parallel transport in the scrape-off layer. Plasma Phys Control Fusion. 2011 Jun;53(6):065004. 